\title{
Reaction times in a task analogous to "same"-"different" judgment
}

\author{
DONALD BAMBER, JOSEPH HERDER, and KATE TIDD \\ Research Service, Veterans Administration Hospital, St. Cloud, Minnesota 56301
}

\begin{abstract}
Subjects responded "yes" if two equal-length strings of letters contained a common letter in a common position; otherwise they responded "no." Thus, the task was to judge whether all or not all of the letters in one string differed from the letter occupying the corresponding position in the other string. Conversely, in "same"-"different" judgment, the task is to judge whether all or not all of the letters in one string match the corresponding letter in the other string. Thus, common-letter judgment and "same"."different" judgment are symmetrically related with "no" analogous to "same" and "yes" analogous to "different." The response "same" is often faster than the response "different." However, in the common-letter task, "no" was slower than "yes." More specifically, both the "yes" and "no" reaction times were consistent with a serial self-terminating search. This is precisely what would be expected from Bamber's (1969) two-process model.
\end{abstract}

One of the puzzling findings in perceptual research has concerned "same"." different" judgments. In a "same".-"different" judgment task, the subject is presented with two stimuli and is required to indicate, by making one of two alternative responses, whether the two stimuli are "same" or "different." Logically, it is necessary to examine every feature of two stimuli in order to determine that they are "same." However, it is not necessary to examine every feature of the two stimuli to deternine that they are "different." Consequently, one might expect "same" responses to be slower than "ditferent" responses. Thus it is surprising that a number of studies have found the opposite. For example, Nickerson (1965) found that, when subjects judged whether two successively presented letters were "same" or "different," "same" responses were faster than "different" responses.

In some "same"." different" studies, the population of stimuli has been constructed in such a way that the individual stimuli vary over several stimulus dimensions. "Different" responses to a pair of stimuli tend to be faster the greater the number of dimensions with respect to which the two stimuli differ. Two stimuli may be said to be minimally "different" if they differ with respect to only one dimension and to be maximally "different" if they differ with respect to every dimension. A number of studies (e.g., Egeth, 1966; Hawkins, 1969; Nickerson, 1967b) have found "same" responses to be faster than responses to minimally "different" stimuli. Occasionally, "same" responses may even be faster than responses to maximally "different" stimuli; however, such a finding is unusual. Following the reasoning given above, one

The authors thank Teresa Kucala and Helen Buerkle for typing the manuscript and David Plantenbers for preparing the figures. This paper is a product of St. Cloud VAH Research Project 0030-02. would have expected "same" responses to be slower than responses to minimally "different" stimuli. Thus, it is surprising that the opposite has been found.

Not all studies of "same"-"different" judgment have found "same" responses to be faster than "different" responses. In fact, a number of variables have been found to influence the relative speeds of "same" and "different" responses. By manipulating these variables, it has been found possible to virtually eliminate or even reverse the difference in speed between "same" and "different" responses. Some of these variables are: probability that the two stimuli are "same" (Downing, 1970), conditional probability that the two stimuli are "same" given the identity of the first stimulus (Thomas, 1974), stimulus codability (Bindra. Donderi, \& Nishisato, 1968), familiarity of stimulus orientation (Egeth \& Blecker, 1971), number of components comprising each stimulus (Silverman, 1973). distance of the stimuli from the fovea (Lefton \& Haber, 1974), and interstimulus interval (Posner \& Boies, 1971).

In summary, "same" responses have often (but not always) been found to be faster than "different" responses. This finding poses a problem because it is contrary to theoretical expectations.

In order to further investigate why "same" responses are often faster than "different" responses, Bamber (1969) performed a study in which subjects indicated whether two horizontal strings of letters were physically "same" or "different." On each trial, the two strings were presented one after the other and always contained equal numbers of letters. Reaction time (RT) was measured from the onset of the second string to the subject's response. Over trials, each string could contain $1,2,3$, or 4 letters. The two strings could either be "same" or could differ with respect to anywhere from one letter to the maximum possible number of letters. 
It was decided to see whether the results of this experiment could be adequately described by a model called the serial self-terminating model (Egeth, 1966). According to this model, the subject compares each letter in the second string with his memory of the corresponding letter in the first string. These comparisons are performed one at a time in sequence. The subject responds "different" as soon as any pair of letters are found not to match. If all pairs of corresponding letters are found to match, then he responds "same."

This model was first fit to the RTs of the "different" responses only. The serial self-terminating model described the "different" RTs rather well (Bamber, 1969, Figure 1). However, using the parameter values obtained from the "different" RTs, the model tit the "same" R'Ts very poorly. Contrary to the model, the "same" responses were faster than the "different" responses.

\section{Two-Process Models}

In order to explain his findings, Bamber (1969) proposed a two-process model. The two components of this model are a serial processor and an identity reporter. These two components operate simultaneously. The serial processor can initiate both "same" and "different" responses. Its operation is described by the serial self-terminating model. Whenever the identity reporter is presented with two matching stimuli, it emits a signal which initiates a "same" response. When presented with two stimuli that do not match, the identity reporter does not emit any signal at all. Both the serial processor and the identity reporter are capable of initiating "same" responses. However, since the identity reporter is assumed to be faster than the serial processor, "same" responses are generally initiated by the identity reporter. Since the serial processor is capable of initiating "different" responses but the identity reporter is not. "different" responses are always initiated by the serial processor. This explains both why the "different" responses conform to the serial self-terminating model while the "same" responses do not and why "same" responses tend to be faster than "different" responses.

In the original version of this two-process model (Bamber, 1969), it was simply assumed that the identity reporter emitted a signal whenever it was presented with two physically identical letter strings. In a subsequent study (Bamber, 1972), subjects judged whether two strings of letters were nominally identical. The results of this study indicated that the identity reporter must be able to signal nominal identity as well as physical identity. (For a discussion of the difference between physical and nominal identity and the effects of this variable on "same""different" RT, see Posner and Mitchell, 1967.)

A number of other authors have also proposed various types of two-process models (Hock, 1973;
Krueger, 1973; Lindsay \& Lindsay, 1966; Reed, 1973, pp. 60-61; Silverman, 1973; Smith \& Nielsen, 1970; Tversky, 1969; Marcel, Note 1). Unlike Bamber's (1969) model, all of the above models (with the possible exceptions of Hock's and Smith and Nielsen's models) assume that the two processes underlying "same" or "different" responses operate in sequence. Thus, the process mediating "same" responses occurs first and is followed by the process mediating "different" responses.

\section{Conjunctive and Disjunctive Matching Tasks}

"Same"-"different" judgment tasks may be termed conjunctive matching tasks. It is proposed to test the two-process model using a disjunctive matching task. The discussion below concerning conjunctive and disjunctive matching tasks is essentially due to Nickerson (1967a, 1973).

In the Bamber $(1969,1972)$ "same".-"different" studies, the subject first examined one letter string. Then he was presented with a second letter string and had to decide whether the two strings were "same" or "different." Let the string presented first be termed the criterion string and the string presented second be termed the test string. Now, in a "same"." different" judgment task, the criterion string defines a conjunctive criterion. Suppose, for example, that the criterion string is FND. 'T hen the conjunctive criterion defined by this string is: ( $F$ on the left) and ( $\mathrm{N}$ in the middle) and ( $\mathrm{D}$ on the right). If the test string satislies this criterion, then the subject responds "same"; otherwise he responds "different." Thus, the "same".-"different" task may be called a conjunctive matching task.

Now, consider a task in which the subject has to decide whether or not the criterion string and the test string contain a common letter in a common position. If they do, he responds "yes"; otherwise he responds "no." This task, which is called the common-letter task, is an example of a disjunctive matching task. Once again, suppose that the criterion string is FND. The disjunctive criterion defined by this string is: ( $F$ on the left) or ( $\mathrm{N}$ in the middle) or (D on the right). If the test string satisfies this criterion, the subject responds "yes"; otherwise he responds "no."

Note that there is a symmetric relation between conjunctive matching tasks and disjunctive matching tasks. In the "same"."different" task, the subject compares each letter in the test string with the letter occupying the corresponding position in the criterion string. If all such pairs of letters are "same," he responds "same." However, if not all letter pairs are "same," he responds "different." Similarly, in the common-letter task, pairs of corresponding letters must be compared. If all the letter pairs are "different," the subject responds "no." However, if not all the letter pairs are "different," then he responds "yes." Thus, in the "same"." "different" task, the subject determines whether all or not all of 
the letter pairs are "same." In the common-letter task, the subject decides whether all or not all of the letter pairs are "different."

The terminology employed in this paper is designed to reflect this symmetry between conjunctive and disjunctive matching tasks. The "same" response in the "same"." different" task and the "no" response in the common-letter task will sometimes be called the "all" response. Similarly, the "different" response in the "same"."different" task and the "yes" response in the common-letter task will sometimes be called the "not all" response.

In the experiment reported here, the subjects judged whether or not two strings of letters contained a common letter in a common position. On each trial, either the two strings were "different" at every position or they were "same" at one position and "different" at all the others. If the two-process model is correct, what results should be obtained from this experiment? Since the two strings of letters are never identical, there should be no output from the identity reporter in this experiment. Thus, both the "yes" and the "no" RTs should be consistent with a serial self-terminating model. In particular, the "no" responses should be faster than the "yes" responses.

Suppose, on the other hand, that these results are not obtained. Suppose that the "no" ("all") responses are found to be faster than the "yes" ("not all") responses. This would indicate that "all" responses in general are faster than "not all" responses. Thus, the phenomenon of "same" responses being faster than "different" responses would only be a special case of this more general phenomenon. Moreover, these results would indicate that the two-process model is incorrect, since this model predicts only the special case and not the general phenomenon.

\section{Review of Literature on Disjunctive Matching and Related Tasks}

Before turning to a detailed discussion of the common-letter experiment, some previous studies of disjunctive matching and related tasks will be reviewed. The most relevant study (Silverman \& Goldberg, 1975) appeared in print shortly after an earlier version of this paper was submitted for publication. These investigators presented simultaneously a pair of four-digit numbers to their subjects. The subjects were required to indicate whether or not the two numbers contained a common digit in a common position. On half the trials, the two numbers contained no common digit. On the other trials, the two numbers could contain $1,2,3$, or 4 common digits in common positions.

If the two-process model is correct, then the results of Silverman and Goldberg's experiment should be correctly described by a serial self-terminating model. Let $L$ denote the number of digits in each number (fixed at four in Silverman and Goldberg's study) and let $C$ denote the number of common digits $(C=0,1$, $2,3,4)$. For tixed values of $L$ and $C$, the serial self-terminating model predicts the expected number of digits that the subject will process before responding. Let this number be denoted by $N(L, C)$. For $L$ fixed at four and for $C$ varying from zero to four, Silverman and Goldberg found that mean RT was almost perfectly correlated $(r=1.000)$ with $N(L, C)$. Thus, a serial self-terminating model described Silverman and Goldberg's results quite well. In particular, negative responses were slower than positive responses, as predicted by such a model.

Nickerson and Pew (1973, Task I) presented their subjects successively with a pair of letter strings. The subjects judged whether the set of letters in the first string overlapped the set of letters in the second string. This set-overlap task is like the common-letter task except for the crucial difference that the ordering of letters within strings is irrelevant. Thus, the set-overlap task is not a true disjunctive matching task. Nickerson and Pew found that negative responses in the set-overlap task were slower than positive responses.

Next, consider two studies of disjunctive matching performed by Nickerson (1967a) and Marcel (Note 1). In these studies, the stimuli could vary along three dimensions. In Nickerson's study, the dimensions were shape, color, and size; in Marcel's study, the dimensions were shape, color, and stripe orientation. On each trial, the subject was given a (verbal) disjunctive criterion. For example, in Nickerson's study, the criterion might have been large or red or circle. Next, the subject was shown a stimulus. If the stimulus agreed with the criterion with respect to one or more dimensions, then the subject made the "yes" response; otherwise, he made the "no" response. Consider only the trials where all three dimensions were relevant (i.e., were mentioned in the criterion). Nickerson found that "no" responses were faster than "yes" responses on trials where the stimulus satisfied the criterion on only one dimension. Marcel found the opposite. Thus, Marcel's results are consistent with a serial self-terminating model and Nickerson's are not. How'ever, the proportion of incorrect "no" responses was quite high in Nickerson's study (over $30 \%$ in one condition). Had the error rate been lower in Nickerson's study, his results might have agreed with Marcel's.

Finally, a study by Sekuler and Abrams (1968) may be noted. These investigators presented their subjects simultaneously with a pair of 4 by 4 matrices. Each cell in each matrix could be either black or white. The subjects indicated whether or not any pair of cells occupying corresponding locations in the two matrices were both black. For complex matrices (i.e., matrices containing four black cells), positive responses were faster than negative responses. In a similar type of experiment, Derks (1972) has obtained a similar 
result. However, Sekuler and Abrams' task is not a true disjunctive matching task; thus, its relevance to present concerns is limited. In a true disjunctive matching task, the subjects would have judged whether any pair of cells in corresponding locations in the two matrices were both the same color (i.e., both black or both white).

\section{The Common-Letter Experiment}

In the experiment described here, the subjects were presented successively with two strings of letters which contained equal numbers of letters. The subjects responded "yes" or "no" according to whether the two strings contained a common letter in a common position. On each trial, the two strings each contained two, three, or four letters. The two strings contained either one letter in common or none.

If Bamber's (1969) two-process model is correct, then the results of this common-letter experiment should be correctly described by the serial self-terminating model. (As previously, let $\mathrm{L}$ denote the number of characters per string and let $C$ denote the number of common characters.) What does the serial self-terminating model predict? First, graphs plotting mean RT vs. L should be linear both for "yes" responses and for "no" responses. Second, it follows from a well-known argument (Sternberg, 1966) that the slope of the RT-vs.-L graph for "no" responses should be twice as great as the slope for "yes" responses. These are the two crucial predictions that are tested in the common-letter experiment.

There are three primary differences between Silverman and Goldberg's (1975) common-digit study and the common-letter study presented here. First, Silverman and Goldberg held L constant at four and varied $\mathrm{C}$ from zero to four. In the present study, C could equal only zero or one and $\mathrm{L}$ was varied from two to four. Second, Silverman and Goldberg presented their stimuli simultaneously, whereas stimuli were presented successively in the present study. Third, Silverman and Goldberg's subjects searched through two strings looking for physically matching digits, whereas the subjects in the present study looked for nominally matching letters.

\section{METHOD}

\section{Subjects}

The subjects were three employees and two voluntary (i.e., unpaid) workers at the St. Cloud VA Hospital. They participated in the study in lieu of their regular work. All subjects volunteered for the study and were below the age of 30 . All were male except Subject S5. All were right-handed except Subject S2.

\section{Apparatus}

The apparatus was a three-tield tachistoscope. Further details are given in Bamber (1972).

\section{Stimuli}

Over the course of the experiment, each subject saw a criterion string and a test string containing two letters each on 256 trials. three letters each on 144 trials, and four letters each on 256 trials. On half of these trials, the criterion string and the test string contained no common letter and, on the other half, they contained exactly one letter in common. This common letter always occupied identical positions in the two strings. It appeared in all string positions with equal frequency. These 656 pairs of criterion string and test string were presented to each subject in a random order.

Criterion and test strings were constructed in the same manner as in the previous study of nominal identity judgment (Bamber, 1972). The letters comprising the two strings were drawn from a set of 12 consonants. The criterion string was constructed from lowercase typewritten letters. The test string was constructed from photofacsimilies of hand-drawn letters. Thus, when the criterion string and the test string contained a common letter, these two letters were nominally identical but not physically identical. Each hand-drawn letter had an uppercase and a lowercase form which were used equally otten. The case (i.e., upper or lower) of each letter in the test string was independent of the cases of the other letters in the string. The letters used to construct the criterion strings and test strings are illusirated in Figures 1 and 2 , respectively, of Bamber (1972). Further details on the construction of stimuli are also given in that article.

\section{Procedure}

On each trial, the subject inspected the criterion string outside the tachistoscope for as long as he wished. Then he rested the fingers of each hind on the two response keys and looked inside the tachistoscope. Upon being signaled "ready" by the experimenter, the subject fixated the center of the darkened ficld and pressed a footswitch. Two hundred milliseconds later, the test string appeared in the field. The subject's task was to press the "yes" key it the criterion string and the test string contained a common letter in a common position, and to press the "no" key otherwise. Subjects S1, S2, and S3 pressed the "yes" key with the dominant hand, while Subjects S4 and S5 pressed it with the nondominant hand. After responding, the subject was informed of whether he was correct or not. He was instructed to maintain a low error rate but to respond as rapidly as possible within that constraint. His RT was measured to the nearest millisecond from the onset of the test stimulus to his manual response.

In previous "same"." different" studies (Bamber, 1969, 1972; Bamber \& Paine, 1973), the test string had been exposed to the subject for only $100 \mathrm{msec}$. Subjects in these studies were able to maintain a low error rate. However, in pilot testing for the present common-letter study, it was found to be quite difficult to respond accurately when the test string was exposed so brietly. Consequently, in the present study, the test string remained exposed until after the subject responded.

According to the time available to them, the subjects were allowed to take from two to four sessions to complete the 656 test trials of the experinent proper. Before beginning these test trials. they were given a session of 120 practice trials. At the beginning of each experimental session, they were given an additional 12 practice trials. All of these practice trials employed stimuli not used in the experiment proper.

\section{RESULTS AND DISCUSSION}

In the following, when an estimate of a quantity is stated in the form $m \pm s, m$ is the estimate and $s$ is its standard error. In all cases, statistical tests are performed by computing a $z$ score and referring to a table of the normal distribution.

\section{Comparison with "Same"-.Different" Results}

As previously, let $L$ denote the number of letters in the test string. The mean R'Ts of "yes" and "no" 
responses in the common-letter study are shown as a function of $L$ on the left side of Figure 1. Shown for comparison on the right side of Figure 1 are the mean RT's from the corresponding conditions of a previous "same"."different" study (Bamber, 1972) in which subjects judged whether or not the criterion and test strings were nominally identical. (These corresponding conditions are the "same" condition and the "different" condition with exactly one "different" letter in the test string.) Note that the RTs in the common-letter study are considerably longer than the $\mathrm{RT}$ s in the nominal-identity study. Moreover, in the nominal-identity study, "all" responses are faster than "not all" responses, whereas the reverse is true in the common-letter study. Finally, in the nominalidentity study, the "same" and "different" RT-vs.-L curves have roughly equal slopes. However, in the common-letter study, the slope of the "no" curve is much greater than the slope of the "yes" curve. So, despite the symmetry between the "same"." different" and common-letter tasks, the results obtained from these two tasks are highly divergent.

The left side of Figure 2 shows, for the conmon-letter study, the proportions of false "yes" responses and false "no" responses as functions of $L$. (A false " no" response is a response where the subject responded "no" when he should have responded "yes.") For comparison, the right side of Figure 2 shows the proportions of false "same" and false "different" responses for the corresponding conditions in the previous nominal-identity study (Bamber, 1972). Thus, in both studies, the proportion of false "all" responses was greater than the proportion of false "not all" responses.

\section{Best Fitting Straight Lines}

Next, the "yes" RT curve and the "no" RT curve for each of the five subjects were tested for linearity. Of these 10 curves, only one deviated significantly trom linearity at the $\mathrm{p}<.05$ level. Consequently, straight lines were tit to each of these RT curves. Table 1 gives the slope and the $L=2$ intercept of the best titting straight line for both the "yes" and the

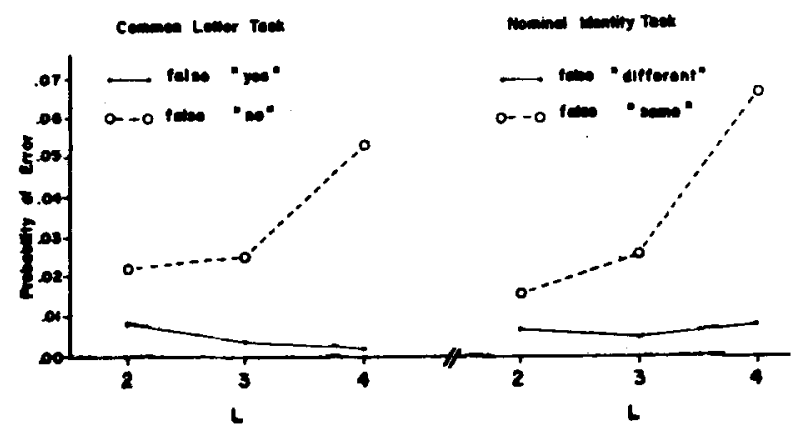

Figure 2. Error rate as a function of L. Left: Comenon-letter task. Right: Nominal-Identity task (Bamber, 1972).

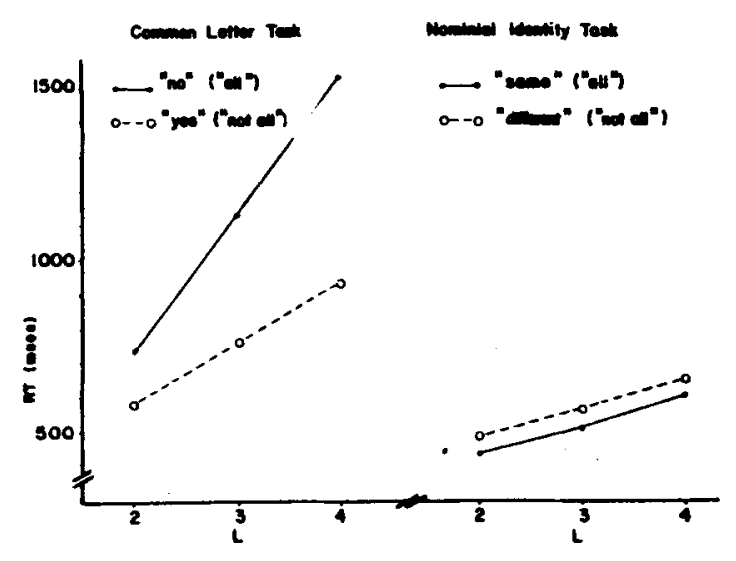

Figure 1. Mean RT as a function of L. Left: Common-letter tack. Right: Nominal-identity task (Bamber, 1972).

"no" RTs of each of the five subjects. (The $L=2$ intercept is the height of the best fitting straight line at $\mathbf{L}=2$.)

If a serial self-terminating model is correct, then it fullous from a well-known argument (Sternberĝ, 1966) that the slope of the "no" RTs should be twice the slope of the "yes" RTs. The last column of Table 1 gives the ratio of the "no" slope to the "yes" slope. For Subjects S1, S2, S3, and S4, this ratio is approximately 2.00 . There is no significant difference between the "no" slope and twice the "yes" slope for any of these four subjects. However, a highly significant difference exists for Subject SS, the ratio of the two slopes being 3.00 .

It is proposed that all of the subjects in this study compared the letters in the test string with the corresponding letters in the criterion string in a serial self-terninating manner. However, unlike the other subjects, Subject S5 often reexamined the test string. The evidence for this is as follows. Subject SS had an extremely low error rate. During the course of over 600 trials, she made only 2 errors, whereas the other subjects made from 10 to 25 errors apiece. Almost $90 \%$ of these errors consisted of responding "no" when the correct respunse was "yes." Evidently, the subjects sometimes failed to detect that a letter in the test string matched the corresponding letter in the criterion string. One way to reduce the number of

Table 1

Intercepts and Slopes (in Milliseconds) for "No" and "Yes" Responses Plus "No"/ "Yes" Slope Ratios

\begin{tabular}{ccccccc}
\hline & \multicolumn{2}{c}{$L=2$ Intercept } & \multicolumn{3}{c}{ Slope } \\
\cline { 6 - 7 } \cline { 5 - 6 } Subject & "No" & "Yes" & & "No" & "Yes" & Ratio \\
\hline S1 & $794 \pm 16$ & $656 \pm 15$ & $375 \pm 17$ & $199 \pm 18$ & 1.88 \\
S2 & $879 \pm 16$ & $680 \pm 14$ & $334 \pm 16$ & $147 \pm 14$ & 2.27 \\
S3 & $689 \pm 13$ & $487 \pm 14$ & $252 \pm 13$ & $126 \pm 13$ & 2.00 \\
S4 & $613 \pm 13$ & $560 \pm 10$ & $346 \pm 15$ & $169 \pm 11$ & 2.05 \\
S5 & $751 \pm 26$ & $546 \pm 13$ & $656 \pm 29$ & $219 \pm 15$ & 3.00 \\
\hline
\end{tabular}


such errors is to examine the test string a second time whenever a tirst examination fails to detect a match between the test string and criterion string. If Subject $\mathbf{S} 5$ had reexamined the entire test string every time no match was detected, then the "no" slope should have been almost four times the "yes" slope. (The reason that the slope ratio would be slightly less than 4.00 is that rescanning would result in correct "yes" responses on a small portion of trials, thus intlating the "yes" slope.) However, the "no" slope was only three times the "yes" slope. This suggests that Subject S5 reexamined the test string only on those trials when she lacked confidence that a "no" response was correct. Alternatively, she may not have reexamined the entire test string but only those parts for which she lacked confidence.

\section{Position Effects}

In a test string of $L$ letters, let the $L$ letter positions in the string be numbered left to right from 1 to $\mathrm{L}$. Figure 3 show's the mean RT of "yes" responses plotted as a function of the matching letter's left-right position within the test string. The figure shows three separate curves corresponding to $L=2,3$, and 4 . If the subjects examined the letters in the test string from left to right on the majority of trials and from right to left on the remainder, results much like those in Figure 3 would be produced. The mean slope of these thrue RT curves was calculated for each of the subjects. For Subjects S1 through S5, these mean slopes were $213 \pm 24,210 \pm 15,169 \pm 17,187 \pm 13$, and $276 \pm 18 \mathrm{msec}$, respectively. This finding, that the mean RT of the "yes" response is highly dependent upon the left-right position of the matching letter, provides further evidence in support of the serial self-terminating model.

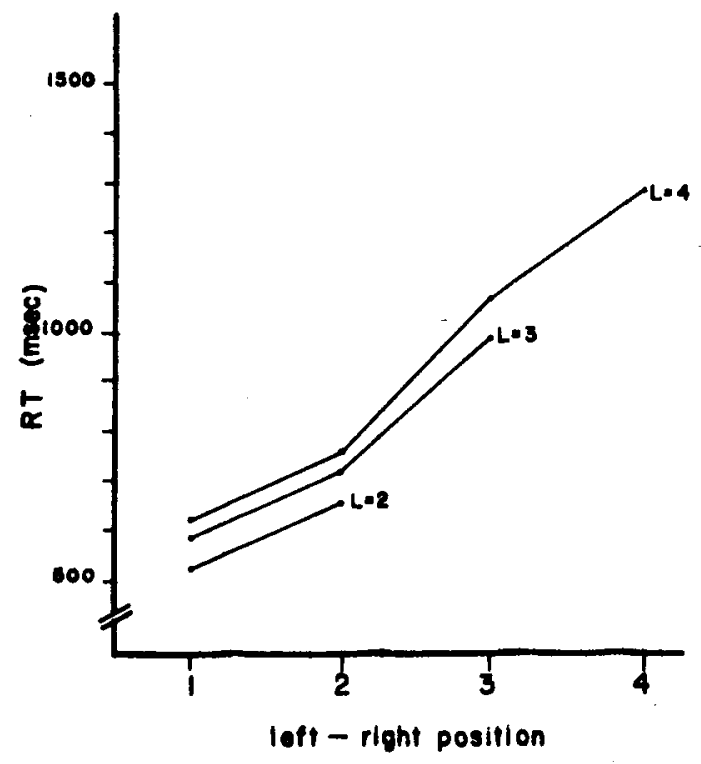

Figure 3. Mean "yes" RT as a function of the left-right position of the matching letter in strings of two, three, and four letters.

\section{Eye Movements}

The eye movements of the subjects in this study were not monitored. However, it is proposed here that during the serial self-terminating examination of the letters in the test string, the subject fixated each letter as he examined it. The evidence for this is as follows. First. unlike previous studies (Bamber, 1969, 1972; Bamber \& Paine, 1973), which employed a 100-msec stimulus exposure, the test string remained exposed until after the subject responded in the present study. The amount of time which the subject devoted to each letter in the test string may be estimated by multiplying by two the RT-vs.-L slope for "yes" responses. Taking the values from Table 1 , the time devoled per letter is found to range from 252 to 4.38 msec over the tive subjects. These times per letter are sulticiently long to allow the subject to tixate each letter in the test string (Woodworth \& Schlosberg, 19.54. pp. 504-507).

The second piece of evidence indicating that each letter was fixated is the following. Suppose the subject did not change fixation. Consider the time taken to respond "no" to a test string containing $L$ letters. This time should consist of the time taken to examine $L$ fulcrs plus various residual times (i.e., sensory nerve tälısmission time, time taken to determine which key to press according to the instructions, motor nerve transmission time, muscle activation time, etc.). Thus the mean RT of "no" responses, should be a linear function of $\mathrm{L}$. Let the height of this straight line at $L=0$ be termed the $L=0$ intercept for "no" responses. The $\mathrm{L}=0$ intercept should equal the sum of the residual times and, therefore, should be positive. The $\mathrm{L}=0$ intercept may be culculated from Table 1 by subtracting twice the RT slope from the $L=2$ intercept. When this is done, it is found that Subjects S4 and S5's $L=0$ intercepts for "no" response's are negative. Moreover, Subject Sl's L $=0$ intercept for "no" responses, while positive, is quite small ( $4+$ msec).

At lirst encounter, this result seems highly embarrassing for the serial self-terminating model. However, the result is easily explained by the following assumptions. First, each letter in the test string is tixated. Second, at display onset, the subject's tixaltion point is sufficiently close to some one of the string letters that he need not refixate that letter. Under these assumptions, the time taken for a "mo" response should consist of the time taken to examine $L$ letlers, plus the time taken to change lixation $\mathrm{L} 1$ times. plus the various residual times. Consequently, the $L=0$ intercept should equal the sum of the residual times minus the time for one fixation change. In this way, it is possible for the L - 0 intercept to be negative.

Thus, there is reasonably convincing evidence that the subjects in this study successively tixated each leller as they examined the test string. Two questions should be asked regarding the effects of these eye movemeuts on the subjects performance. 
First. Was the process of common-letter judgment forced to be serial simply because eye movements are serial!" Evidently not. Patterns of RT indicative of scrial processing in a primarily left-to-right direction have been obtained not only from the present common-letter study but also from earlier "same"."different" studies which employed a $100-\mathrm{msec}$ stimulus exposure (Bamber, 1969, 1972; Bamber \& Paine, 1973).

Second, might the eye movements have suppressed a capability of making fast "no" responses? To test this possibility, three additional subjects were brietly tested on the common-letter lask. By using only short strings (two letters each), the exposure time of the test string could be limited to 100 msec. All three subjects responded "no" more slowly than "yes" despite the fact that they all made the "no" response with their dominamt hands. Thus, eye movenents were not the cause of "no" responses being slower than "yes" responses.

\section{CONCLUSIONS}

Bamber (1969) proposed a two-process model of "same"." different" judgment. If this model is correct, then it should be possible to empirically separate the two processes. Previous attempts to do this (Bamber, 1972; Bamber \& Paine, 1973) have liciled. The present experiment and Silverman and Goldberg's (1975) experiment are a new attempt. The results from these two experiments indicate that common-fetter and common-digit judgment are serial self-terminating processes. This tinding indicates that onc of the processes hypothesized by Bamber (the scrial processor) exists and can function independently of the other hypothesized process (the identity reporter). In and of itself, this finding does not provide eridence for the existence of the hypothesized identity reporter. However, in the context of previous lindings (c.g.. Bamber, 1969, 1972), this finding does provide indirect evidence for the existence of the identity reporter.

\section{REFERENCE NOTE}

1. Marcel. A. 1. Sequential and parallel processing and the nature of the decisions in puttem recognition and classijicution. Expanded version (May 1970) of a paper presented at the meeting of the Experimental Psychology Swciety, London, January 190.

\section{REFERENCES}

Baysen, D. Reaction times and error rates for "same". "different" judgments of multidimensional stimuli. Perception \& Psychophysics, 1969, 6, 169-174.

Bumier. D. Reaction times and error rates for judging nominal identity of letter strings. Penception \& Psychophysics. 1972, 12, 321-326.

Bamere, D., \& Paine, S. Information retrieval procesces in "same":" "ditferent" judgments of ketter strin $\mathrm{xs}$. In S. Komblum (Ed.), Altention and performance IV. New York: Academic Press, 1973. Pp. 477-495.

Binden, D., Dokdenu, D. C., \& Nisursato, S. Deciston latencies of "sume" and "different" judgments. Perception
\& Psychophysics, 198, 3, 121-130.

Derks, P. L. Visual recognition of similarity and identity. Journal of Experimental Psychology, 1972, 95, 237.239.

Downing, B. D. Response probabilities and "same"."different" reaction times. Perception \& Psychophysics, 1970, 9. 213-215.

Egeth, H. E. Parallel versus serial processes in multidimensional stimulus discrimination. Perception \& Psychophysics. 1966, 1. 245-252.

Egeth, H., \& Blecker, D. Differential effects of familiatity on judgments of sameness and difference. Perception \& Pyychophysics, 1971, 9(4), 321.326.

Hawkins, H. L. Parallel processing in complex visual discrimination. Perception \& Psychophysics, 1969, 5, 56-64.

Hock. H. S. The effects of stimulus structure and familiarity on same-different comparison. Perception \& Psychophysics, 1973, 14. $413-420$.

KrUeger. L. E. Efiect of irrelevant surrounding material on speed of sump-different judgment of two adjacent letters. Journal of Experimental Psychology, 1973, 98, 252-259.

Lefton, L. A., \& HABER, R. N. Information extraction from different retinal locations. Joumal of Experimental Psychology, 1974. 102. 975-980.

Lindsay, R. K., \& Lindsay, J. M. Reaction time and serial versus parallel information processing. Journal of Experimental Psychology, 1966, 71, 294-303.

Nickerson, R. S. Response times for "same".-"different" judgments. Perceptual \& Motor Skills, 1965, 20, 15-18.

Nickerson, R. S. Categorization time with categories defined by disjunctions and conjunctions of stimulus attributes. Jounal of Experimental Psychology, 1967, 73, 211-219. (a)

Nickerson, R. S. "Same"-"different" response times with multiattribute stimulus differences. Perceptual \& Motor Stills, $1967,24.543-554$. (b)

Nickenson, R. S. The use of binary-classification tasks in the study of human information processing: A tutorial survey. In S. Kornblum (Ed.), Attention and performence $\boldsymbol{I V}$. New York: Academic Press, 1973. Pp. 449-475.

Nickerson. R. S., PEw, R. W. Visual pattern matching: An investigation of some effects of decision task, auditory codability, and spatial correspondence. Joumal of Experimental Psychology. 1973, 98, 36-43.

Posner, M. 1., \& Bores, S. J. Components of attention. Psychological Review, 1971, 78, 391-408.

Posner, M. 1., \& Mitchell, R. F. Chronometric analysis of classification. Psychological Review, 1967, 74, 392-409.

ReEd. S. K. Psychological processes in pattem recognition. New York: Academic Press, 1973.

Sexuler, R. W., \& Abrams, M. Visual sameness: A choice time analysis of pattern recognition procesces. Joumal of Experimental Psychology, 1968, 77, 232-238.

Silverman, W. P. The perception of identity in simultaneously presented complex visual displays. Memory \& Cognition. $1973,1,459-466$.

Silverman, W. P., \& Goldberg. S. L. Further confirmation of same vs. different processing differences. Penception \& Psychophysics, 1975, 17, 189-193.

Smitr, E. E.. \& Niztsen, G. D. Representations and retrieval processes in short-term memory: Recognition and recall of faces. Joumal of Experimental Psychology, 1970, 85, 397.405.

SteRnBerg, S. High-speed scanning in human memory. Science. 1966, 153, 652-654.

Thomas, E. A. C. The selectivity of preparation. Psychological Review. 1974, 81, 442-464.

Trersky, B. Pictorial and verbal encoding in a short-term memory task. Perception \& Psychophyaics, 1969, 6, 225-233.

Woodworth, R. S., \& Sctilosbenc, H. Experimental puxchology (rev. ed.). New York: Holt, Rinehart \& Winston, 1954.

(Received for publication March S, 1975; revision received July $7,1975$. 